\title{
Pursuing resilience in architectural design through international experimental projects: exploring new boundaries in the design studio pedagogy.
}

BASSANESE, S., RODEGHIERO, B. and ESPANYOL, A. 


\title{
Pursuing resilience in architectural design through international experimental projects: exploring new boundaries in the design studio pedagogy
}

\author{
Silvia Bassanese, Benedetta Rodeghiero, Aida Espanyol
}

\begin{abstract}
In response to the current global crisis, there is a growing demand for responsible behaviour in designing and building that can accommodate user needs through the design process. This chapter describes an innovative approach to the design process aiming to generate a model adopted by an international collaboration who are reconsidering the traditional design process and addressing a new paradigm of the thinking process.
\end{abstract}

The project is experimental in nature and discusses the educational frameworks in architecture. It optimises a model, which demonstrates breakthroughs and trend-setting educational approaches and is potentially transferable to a range of other professions. The chapter argues that the educational ethos of `ethic of resilience`should be pursued by pushing the boundaries of the conventional Design Studio towards the formation of adaptive system settings. All the participants at the various stages of the innovative educational framework, named Build Our Nation (BON) and its first application Taifa Letu Tujenge (TLT), have already demonstrated, on one hand to be able to learn from the experience achieved from various stages undertaken in the past, and, on the contrary, to be flexible enough to proceed with changes reflecting on the external conditions.

The vision is that the Higher Educational Institutions and, especially, universities must become more co-productive actors in society. It can be useful to think of a university as a manufacturer; and subsequently, a manufacturing company as an advanced workshop; a workshop as a real- world project; therefore, a real-world project connoted back to the meaning of university. This vicious cycle of pedagogy embedded in learning and teaching should be central to any higher education focusing on design and research aiming to inform each other through the values of social capital.

Keywords: system thinking; breakdown of settings; practice research; ethics; social capital. 
Introduction

Social capital is the desired collective or economic benefits achieved by the preferential treatment and cooperation between individuals and groups. (Coleman, 1988). Investigation of social capital cannot, however, be regarded as the only path of resolution for all adverse conditions of this world; but there is enough evidence to illustrate that to some extent investment in research on social capital can play a significant and active role in our society (Woolcock, 2000). Philosopher Galimberti (2009) has pointed out the general nihilism of the young generation in Western society is a more common cultural issue than an individual psychological problem. The experimental international model ‘Build Our Nation’ (BON), described in this chapter, depicts an innovative educational model that allows pragmatic thinking in design and aims at promoting cross- cultural and problem-solving directives through value-guided architectural solutions. BON is an international project by a research group based between the Scott Sutherland School of Architecture and Built Environment at Robert Gordon University in Aberdeen, United Kingdom and the Escola Tècnica Superior d'Arquitectura de Barcelona, Universitat Politècnica de Catalunya in Barcelona, Spain and the Escola Superior d’Arquitectura de Reus, Universitat Rovira i Virgili in Reus, Spain. The group focus on the promotion of innovation in educational processes linking design studio activities. 'Taifa letu tujenge’ (TLT), which means 'build our nation' in Swahili, is the first application of BON to a real project using its framework and methodology.

In all the current discourse concerning the need for know-how in dealing with difficult problems and in confronting radical changes, it has become urgent also in the field of architectural design to explore the potentialities of design thinking deeply. The territory opened by recent publications and papers by Brown (2009); Charnley \& Lemon (2011); and Dorst (2011) suggest the significance of crossdisciplinary engagements, which are transferrable beyond the core of the design disciplines. This chapter argues that the on-going educational model of BON and its first project TLT be already challenging the boundaries of design thinking; thus successfully demonstrating an innovative experimental model of system thinking in architecture.

As one of the original contributors to the third generation systems thinking at the Institute for Interactive Management, Jamshid Gharajedaghi (2011), defines the four foundations of system thinking are: socio-cultural systems, holistic thinking, operational thinking, and design thinking. Gharajedaghi (2011: 88) establishes these foundations by stating:

The depth and beauty of interactive design and the magic of holistic thinking (iteration of structure, function and process) when combined with the power of systems dynamics, create a competent and exciting methodology that goes a long way in dealing with the emerging challenges of our time by responding to the operating principles of openness, purposefulness, multidimensionality, emergent property, and counterintuitive behaviour of socio-cultural systems. 
The frameworks of BON and TLT pull together a group of participants comprising volunteer students, academic members and future users, who collaborate on an international Design Studio and perform interchangeable roles of leadership to develop a chosen real-project. The various activities of TLT are workshops, blogs, performances, exchanges and live events that are mainly led by students in a highly motivated and ethical environment (Figure 1-1). Power is enhanced when it is shared when the individual 'disappears' in a collaborative team group and the students are more likely to implement an idea when they have had hands-on intervention in shaping it

In our liquid modernity, where social forms and relationships no longer have enough time to solidify (Bauman, 2007), the possibility to get involved in a real-world project requires a matter of competence, knowledge and skills, which are some of the best powers of culture. BON and TLT are pushing the boundaries of the conventional mandatory setting of a Design Studio regarding professional ethics and practice. BON and TLT optimise the social capital of architectural education according to the highly sensitive social context of the real project. In pursuing an 'ethic of resilience', crisis and contemporary socio-economic changes can be perceived as ideal opportunities continuously to reset the relationship between people and their environment.
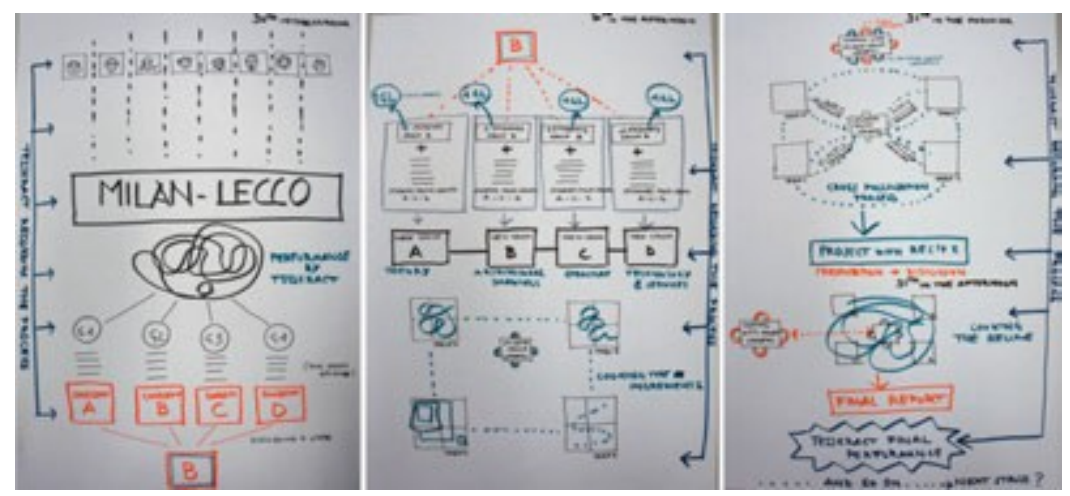

Figure 1-1. Layout of the second stage of TLT (source: www.buildournation.org)

\section{Research In Search of 'Resilience'}

System thinking still represents an unknown territory to be deeply investigated for the simplifying complexity of designing and building processes. It is concerned with seeing through chaos, managing interdependence and understanding choice. Systems thinking allows us to understand how various elements in our life, regarded as systems, relate to each other and influence one another within a whole, such as, eco-systems covering air, water, movement, plants, and animals; and how these relate to each other to sustain our planet (Peter, 1981). Similarly, BON explores the adoption of a whole systems approach to more sustainable and innovative design. This section of the chapter presents a framework of methodological elements: a) the need to identify relationships between parts of the system; b) the requirement for trans-disciplinary skills, and c) the dynamics of a 
flattened hierarchy to ultimately optimise the whole. In BON, two main trajectories are under investigation, both strongly linked to the extra-curricular nature of the actual framework. On the one hand, are the educational contexts with the aim of rethinking design. On the contrary, are the environmental-oriented contexts with the aim to rethinking construction. Based on these objectives, the panel of academic members of BON has identified a set of three target issues:

- Ethical standards and social equity. The project must adhere to the highest ethical standards and sense of responsibility. The aim is to support social equity at all stages, from the design process to the construction.

- Contextual impact and holistic thinking. The project must convey a high standard of architectural quality in the way it addresses cultural and physical values. The aesthetic value must `interact` with the surrounding environment.

- Innovation and transferability of knowledge. The project must demonstrate breakthroughs and trend-setting educational approaches and must be transferable to a range of other applications.

In alignment with the global community commitment to embrace the challenge to reorient itself economically, socially, and ecologically, members of Higher Education have the responsibility to reinvent the teaching, researching, and learning praxis related to the world in which we live and to align with the principles of sustainable development. What is valuable about BON is that it is a process of building a 'resilient' system itself. On one hand, it is 'resilient' because it is adaptive, able to change and learn from experience. On the contrary, it is complex because it is made by multiple and interconnected elements (Holland, 2006). BON is establishing an iterative and generative process: from rethinking design to rethinking construction, back to rethinking design and rethinking construction.

"Because the fundamental problem of architecture and culture is that of freedom", as beautifully explained by Branzi (1975: 10-12), educational programmes must be able to help students in pursuing an 'ethic of resilience' capable of returning to the original shape of the world, as after having been confronted by external forces. That is quite problematic for a design research community that cherishes many oversimplifications of its object of study and delegates many tasks to other disciplines, especially in practice. The vision of BON is `to set up highly equipped students by instilling confidence in their skills as future professionals and by strongly challenging the foundation of their education. The nature of this project brings together volunteer design researchers, who are enthusiastic about challenging themselves and the limits of architecture as a discipline outside of the necessary curricular framework. It represents a unique opportunity within academic education for a wider perspective of architecture in a true mutual learning environment with the outcome of a fuller knowledge of architecture and its position in the world. 
It might be argued that these kinds of goals be very common in the Design Studio. However, the core of BON is fundamentally defined as a big playground founded on some experimental notions of the 'Situationist International' during the 1960s (Andreotti \& Costa, 1996; d`Anjou, 2011). As part of the induction to BON, the first message to the students is: "This is a playground. This is our playground. We all together are the players (Figure 1-2). Once agreed on the rules, the game must go on!” BON and TLT are inaugurated by introducing the methodological ground at the outset, and explaining that every space that is room for gaming requires establishing some limits manifested in prohibitions and opportunities for the players. Consequently, BON becomes a playground, and the players are transformed into inhabitants of a new territory (Iacovoni, 2003).

Because there is a specific ground, there is also an unpredictable transformation under the pressure of the forces that put force on it. In playing through spatial and virtual forms, the playground must continually be redefined, by creating interfaces and/or giving form to a set of rules (Figure 1-3). That implies a demanding process of negotiation and joint decision-making between players. It should also be noted that a competitive but playful context helps students to gain knowledge more convincingly.

The space in TLT, where the game of reality is taking place that is the real project's issues requires the players to improve their critical thinking, competence, flexibility, counter-intuitive behaviour, and many other operating qualities related to system thinking. Consequently, BON includes a pattern of consciousness of how arbitrary the rules and the unwritten are as well as tacitly obeyed conventions. It clearly shows the limits society has drawn for us regarding designing and building and the players have the power to question these rules and, if necessary, to propose new ones. In this process, the emotive challenge of the brief becomes necessary, with the focus on addressing deep social issues through architecture. That highlights the limits but also enhances the ethical approach to architectural designing (Wasserman, Sullivan \& Palermo, 2000) in a way current studio projects are not usually assessed.

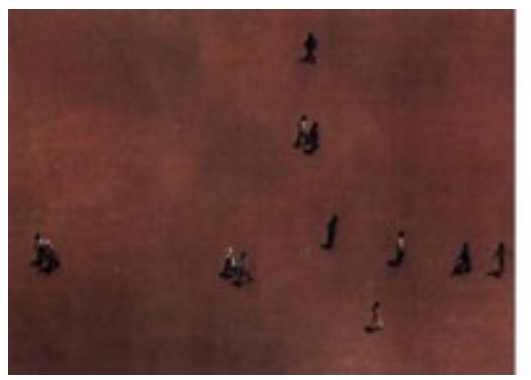

Figure 1-2. From grounds to playgrounds

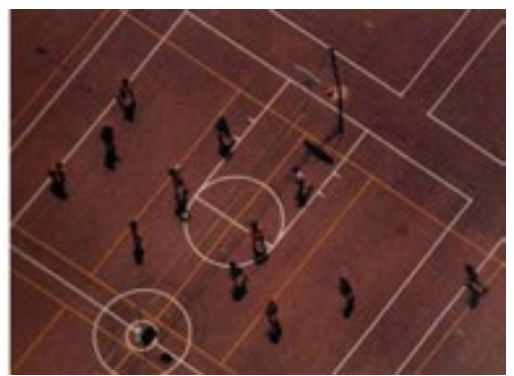



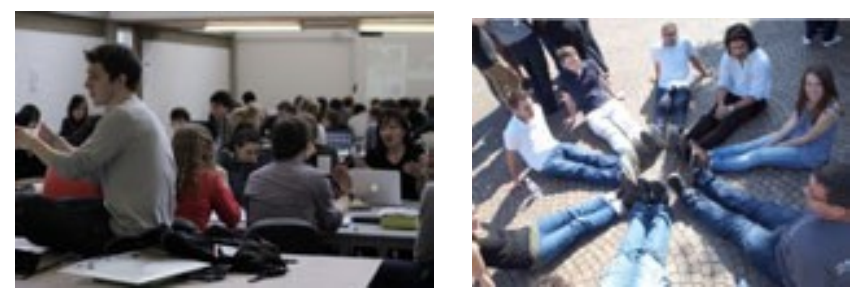

Figure 1-3. Self-formation of teams, playgrounds and rules during TLT (source: www.buildournation.org)

The first application of BON, TLT consists in the real-world project of designing and building a community centre for women in the city of Bukavu, in the Democratic Republic of Congo (DRC), Africa. TLT involves multitudes of students spanning four Schools across Europe. In April 2011, the collaboration was organised by members from these universities with the mutual help of architecture students. The brief declares: “The African nations set up political objectives for the promotion of women's activities. Women have been marginalised for a long time in the DRC. There are many social and professional activities, which do not integrate women. We want to change these dynamics locally by creating a space where development is catalysed by the participation of the women themselves. This will improve the social and economic conditions for women in the surrounding areas and throughout the city. General objectives of the present project propose to facilitate interaction between women from diverse backgrounds - rural, urban and suburban. This will develop an area of fundamental change to help reduce the complex inequalities women face regarding income and opportunities”. In the complexity of the social, political, and economic conditions, this self-built centre will be a catalyst for social change through a participatory and innovative action involving about 1,000 women from the province of Bukavu.

By the very nature of the project, many people from professionals and students can be involved. The four different collaborator Schools of Architecture, Built Environment and Engineering across Europe have worked together having a common effort, ethos and aim of positive participation. Having to deal iteratively with the structure of BON (i.e. the major participants and their relationships), the function (i.e. the specific outcomes); the process (i.e. the know-how and the sequence of activities); and the context (i.e. the role the system plays in its containing environment) collectively imply seeing the whole and understanding it together. It involves a continuous process of re-orientation to fit the rules of the specific playground.

The following section illustrates the modularity of BON. According to Gharajedaghi, a complex product (i.e. TLT but also BON) is made by smaller sub-systems, referred to as the set of distinct but interrelated platforms. Each platform (i.e. the different stages of TLT) hosts a set of specialpurpose modules about structure, function, process and context of the overall project (i.e. TLT does not exist if separated from the framework of BON). As in a system thinking framework, the relationships and the interfaces among platforms must be explicitly defined. In this innovative 
project, the parts operate independently with the ability to be relatively self-controlling and to act as responsible members of a coherent system. Practically and at present, there are two frameworks which are running parallel and constantly crossing each other. On one hand, BON is going to become an international research group aiming to explore new trajectories of innovative participatory learning methodologies. On the contrary, TLT is the first trial project in the framework of BON; and represents an important test for evaluating the results in the light of the general outcomes of this innovative educational experiment.

\section{Challenging Design Studio}

This section describes the structure of BON and TLT. Because both the frameworks run in parallel, sometimes it is not so easy to identify their limits. Also, it is equally difficult to make a clear distinction between the two. This is due to the reasons that, firstly BON is the original organisation that has a structure within the primary matrix; and secondly, it has nothing of the first application i.e. the real-world project TLT is generated without having a framework. Therefore, every part of TLT is correlated with its other parts (i.e. the progressive stages) and establishes the whole of BON and TLT with a direct and close relationship. Through exploring a territory and trying to orient participants, TLT represents exactly the experience of everybody involved in the project and which is shared from his/her specific role with the other participants, and that represents an itinerant movement that would touch the territory of BON as a whole. Knowing the factors that influence the process of the entire system, the design provides participants with the knowledge necessary to efficiently work within, manage and facilitate that process (Figure 1-4). The case study TLT with its operational anecdotes demonstrates those factors.

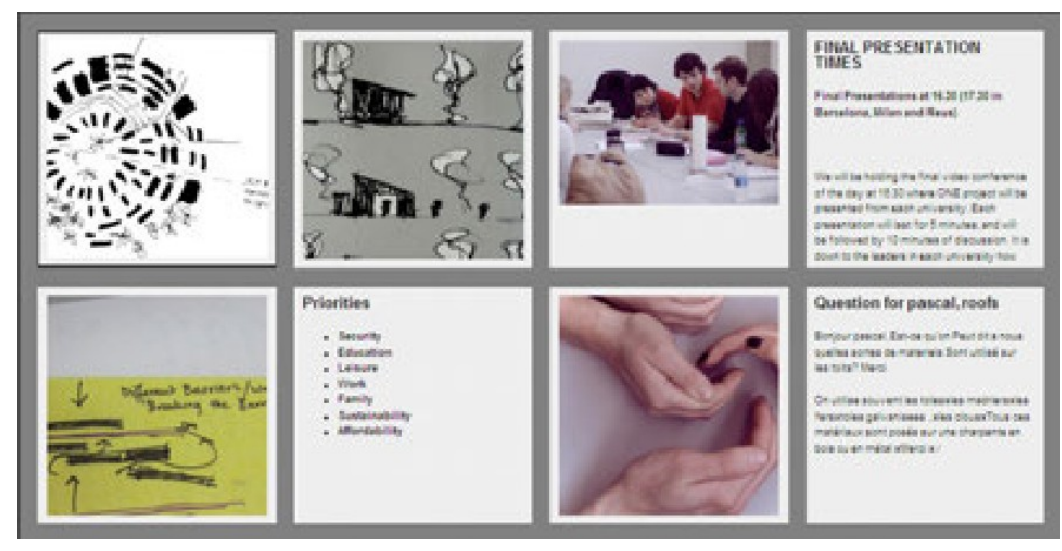

Figure 1-4. Blog (source: www.buildournation.org)

Concerning BON, a panel of academic members of four different European Schools set up a Memorandum of Understanding to deal with the actual and the future real-projects. Some of those academics have created an international research group, which focuses on innovative 
methodological developments in studio teaching. The academic members are invited to take part in the leadership of the various stages of the first real project TLT. Until now, three steps for TLT have been organised, and the fourth is currently underway. Under the supervision of the staff, each stage is arranged by students responsible for leading the events and the activities. The users of the real project, the women of Bukavu in the case study TLT, are involved in the design from the very beginning of the process. There is a permanent exchange of ideas between students and women, which culminates in the final construction of the centre through participatory processes of selfbuilding construction.

The Stages of TLTthat

Stage 1 of TLT involved about 250 students from the four European Schools and was led by a collaboration of architecture students. Each School worked independently in mixed vertical groups of about eight students from their institution. The only common institutional rule was that the students should include members from Year 1 to Masters level to guarantee the cross-pollination of ideas independently from their knowledge. The specific outcome of the first stage was to brainstorm ideas creatively and to synthesise them quickly into visual concepts, done over only one day. The concepts were then finalised into three A2 sheets comprising: the social aspect, the technical aspect and the exhibition graphics (Figure 1-5 and 1-6). The goal of the event was to build a visual dictionary of concepts and information to be used in the following stages. A conference at the end of the day engaged student leaders across Schools to exchange and share initial ideas. Web conferences and a blog were also in place to guarantee continuous media feedback, bearing in mind that the use of IT facilities as common tools for the young generation yields very effective results.

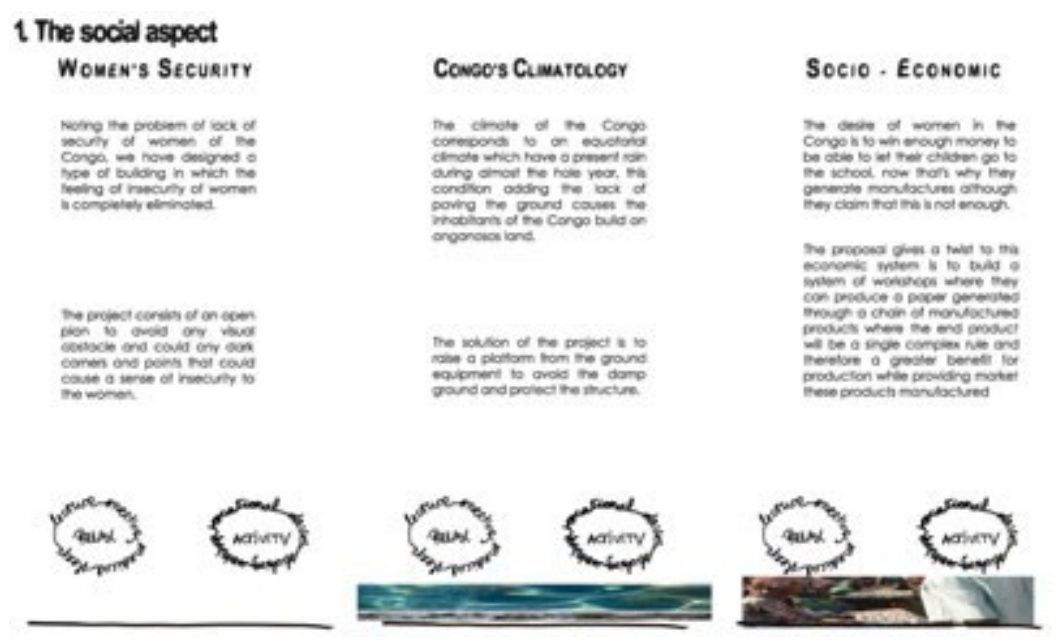

Figure 1-5. Examples of concept by one team group (source:www.buildournation.org) 


\section{The tectnical aspect}

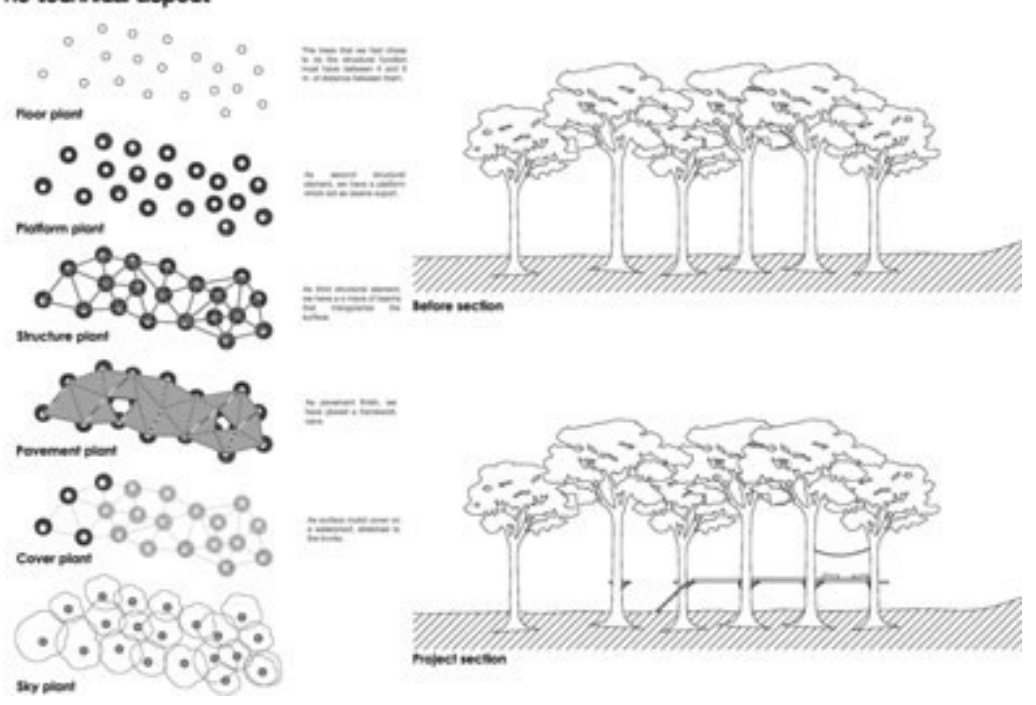

\section{The exhibition graphic}

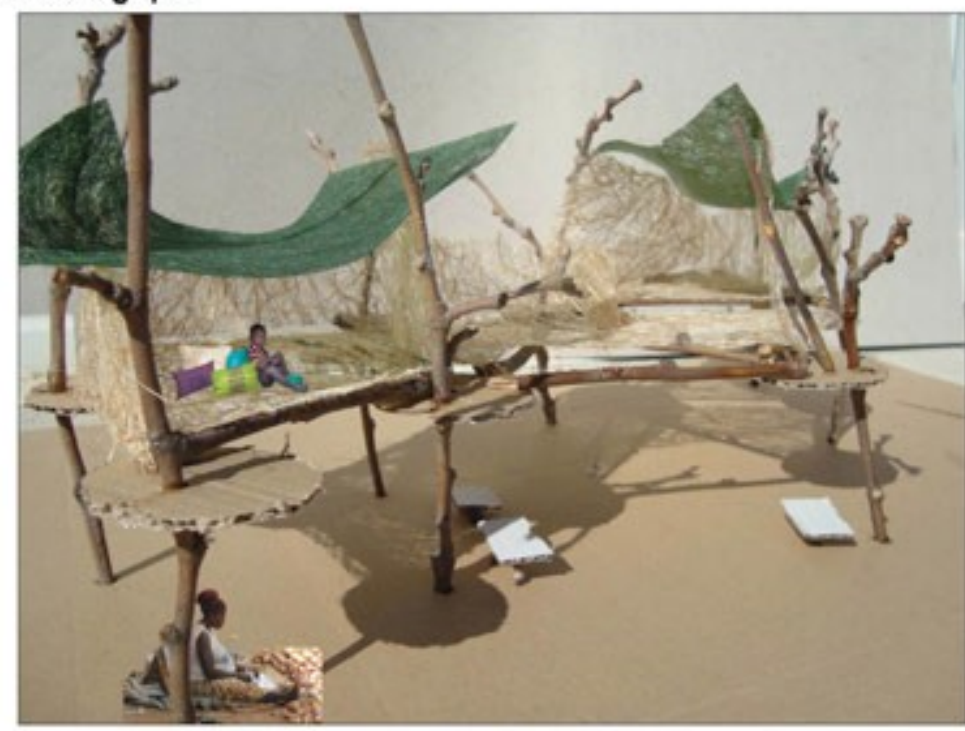

Figure 1-6. Examples of concept by one team group (source:www.buildournation.org)

Understanding requires a period of orientation that forces students to think and, in thinking, to exercise their critical faculties. When large numbers are involved, the groups must always tackle the problem of creating dynamics able to continually re-establish a balance. This equilibrium inevitably changes with the passing of time. Friedman (2000) states that society is deeply grounded in communication and that a utopia can become a reality only if the number of the members in one group does not exceed a 'critical quantity'. Moreover, one of the big problems that the future generation of architects must urgently address is that mass society is expanding everywhere and exercising one's critical faculties becomes increasingly demanding (De Carlo, 2005). BON is not concerned with large numbers, rather patronises a large society of small groups forming and reforming according to the circumstances. This approach can guarantee the iteration of the process not only in the short-term but also in the medium and the long-term. 
Stage 2 of TLT. Between Stage 1 and the commencement of Stage 2, the women of Bukavu have been asked to select one of four concepts. These four concepts comprise only one selected concept from each School that differs from the other three due to the provenience (i.e. independent organisation of the team group working in each School), and the selection process (i.e. autonomous self-selection from the multiple concepts of stage 1 in each School). After a process of self-selection of participants, about 30 students from the four Schools worked together in the same place in a twoday intensive workshop. Four new groups of international students initially investigated further variations of the concept chosen by the women; then, all the students together democratically selected the best variation of the idea; and finally they started brainstorming and exploring through models and drawings the first aspects of the now called project for the centre. At this stage, the twoday activities were led by student leaders chosen alternatively from each group who relied on the progression of the work. The role-play regarding interchangeable-leadership gave them the feeling of being a 'chef' dealing with the quality of the ingredients, the storage of them and the preparation of reports. Outputs of Stage 2 included a final report made mainly of drawings and sketches, and a short video on the collaborative design process, created under the supervision of a professional filmmaker who synthesised the entire effort at this stage (Figure 1-7).

\section{Environment}
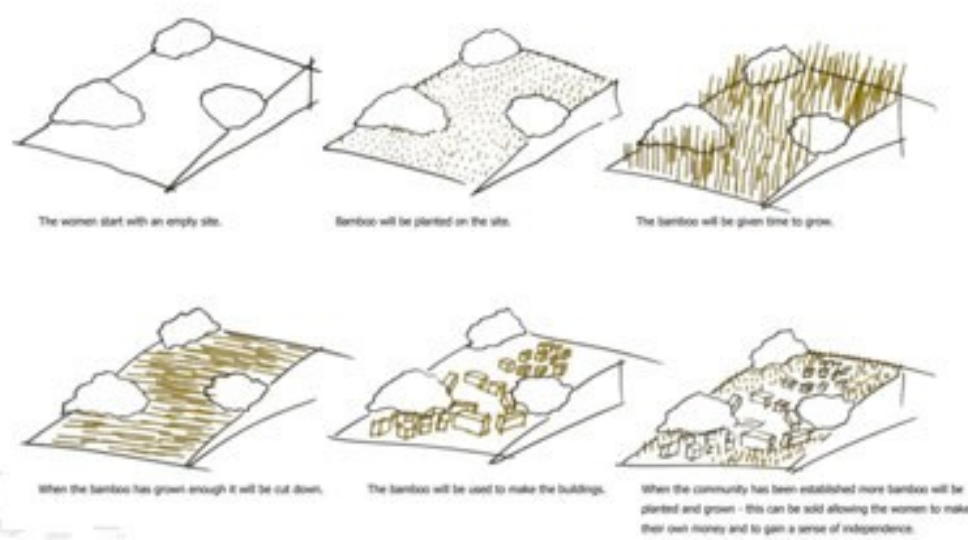

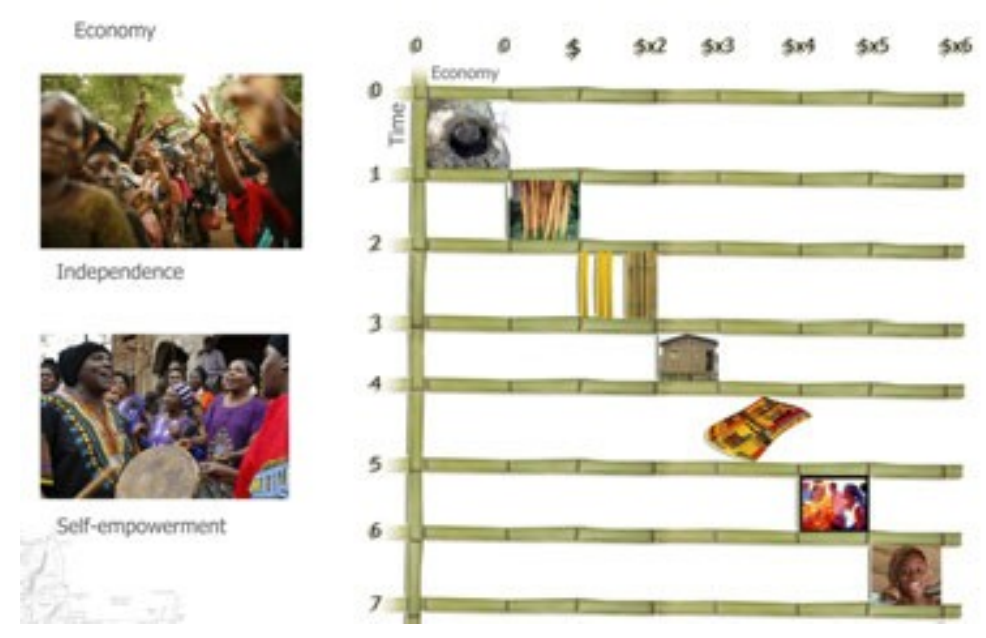

Figure 1-7. Interesting results from the final report (source:www.buildournation.org)

The use of intensive and short workshops enables students to see and experience the work from a multicultural and internationally engaging perspective. The context plays a critical role in defining the degree of influence the system plays within the contained environment. For example, the easyto-understand metaphor of cooking is extensively used to make clear to the students that the whole process is a sequence of well- organised stages; but, one cannot just take the ingredients, no matter how good they are, throw them in a pot, add heat, and wait for a wonder to happen. This process should involve a good choice of recipe that tells one which ingredients and in which order they are to be prepared; and only then will the dish taste good when it is served. Price (2003: 87) remarked beautifully: “One sees architectural responsibility avoiding involvement with the whole process. Certainly one can see it in the resulting products, whether they are poorly designed or badly used, or left to stand around too long [...]. It is like they should last an appropriate time, just like the storage of food, the preparation, the eating, and theevacuation'.

Stage 3 of TLT focused on the economic and procurement aspects, rather than on 'pure design' issues. The emphasis was on feeling and testing the reality of the project with as close a relationship as possible with the women in Bukavu, the real palimpsest of the site, and the environment of DRC. Building up a stronger link and exchange of opinion directly with the last users has become vital because the first two stages were carried on through the linguistic involvement of a mediator. This process involved dealing with non-verbal communication and with limited economic and technologic resources (Rapoport, 1990; Friedman, 2003). Both are challenging playgrounds, where the students face realistic constraints and opportunities. Stage 3 was open to a larger number of students compared to Stage 2 (80 in total, 20 from each School). Six groups of students were organised into new vertical units and focused on one specific task (site factors, social factors, technological factors and legislation factors). The entire process was led by various student leaders, who along with the other students, had to deal with an international architectural studio team with a 
hierarchical structure and to work and to communicate from different locations. The goal was to enhance the general know-how of the actors. It implied 'do it you' activities including interviewing professionals about the specific issues of the project to bring in ever new disciplinaryexpertise as it becomes relevant (Figure 1-8).
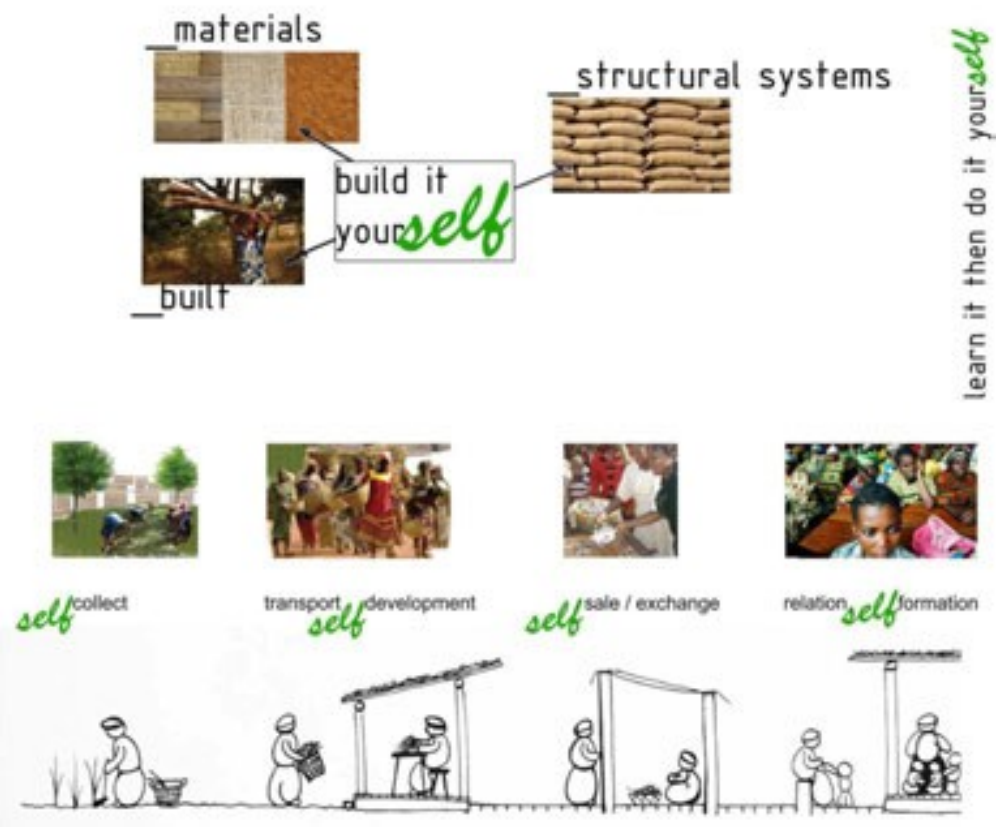

Figure 1-8: Self-building process (source: www.buildournation.org)

The possibility of building the first prototype of one unit of the centre in Bukavu is under investigation. That implies the completion of a manual for self-builders in the light of some relevant examples from the past (Friedman, 1977) to assess the real women`s skills and the student`s ability to use non-verbal instructional drawings for building a small mock- up prototype.

Stage 4 of TLT. The on-going Stage 4 focuses on a participatory approach to design, attempting actively to involve the participants in a real international design studio team. In such a playground the participants have specific roles: architects (i.e. students), experts (i.e. staff members and professionals selected by students before the workshop) and real users (i.e. invited women of Bukavu). The objective is to work intensively together in light of the definition of a 'common ground, a process of negotiation of ideas starting from the results achieved by each university during the autonomous research of Stage 3. This stage culminated in a two-week intensive workshop in the Biennale sessions, the special project of the $13^{\text {th }}$ International Architecture Exhibition, directed by David Chipperfield and proposed for EU funding. The whole process is recorded and illustrated through a web-documentary which includes two parallel frameworks: one on the history of the educational process of BON, and the second on the development of the participatory architectural project of TLT. Part of the web-documentary was filmed in the DRC, where the issues concerning the context, local needs, the challenging participation of the women of Bukavu and some first 
experimental playgrounds between students (real architects) and women (real final users) were covered. The interactive web documentary (ICT-based tool) supports BON and TLT with a powerful communication platform to promote its experience and share its innovations with a broad audience. The entire process was prepared for the Venice $13^{\text {th }}$ International Architecture Exhibition. The objective was to carry on with this project on a daily basis at the venue to share the outcome with the participants of the workshop and the public of the Biennale in general, who then were able to orient and constantly re- orient themselves in the complex territory of BON-TLT, while finding an individual `path` in the flow of the workshop.

The specific outcomes of the stages of TLT must be evaluated about the general goals of the frameworks of BON. They have been previously mentioned and identified three target issues: ethical standards and social equity, contextual impact and holistic thinking, and innovation and transferability of knowledge. The conventional rule adopted by the Western professionals underpinned in the ideology that 'any decisions must be culture-specific', turns out to be negative after being assessed in a 'post-occupancy' evaluation. That often happens because of the complex relationships among cultures, behaviours and the built environment. As the territory should be considered a palimpsest, being the results of many overlapping processes and stratifications (Corboz, 1985), there aresome important social events, footprints, traces, and signs that constitute vital `latent functions` for designing a new building in its context. In fact, contemporary architectural practice, which tries to identify and allocate the proper time to decipher and to understand and absorb these kinds of `latent functions`, is considered a gadget. BON aims at a general awareness of that problematic issue, which means one additional step in improving the ethical approach to architecture and the built environment. For over ten years Rapoport (2009) has asserted that architecture should be considered a science-based profession, which is concerned with problem-solving rather than a purely artistic activity. Also, one of the principal purposes of rethinking design and building is to create users-oriented environments to respond to their particular culture. This objective represents the common ground for both BON and TLT. Rapoport strongly highlights that designers have to be a kind of 'surrogate for users'. Similarly, BON and TLT encourage the philosophy that architecture must adapt to people, and not the contrary.

First results: Impact on Architectural Education

The framework of BON and TLT suggest that the process, use of different combinations of certainty, chance, and choice rather than the initial condition, is mostly responsible for future conditions. This implies that understanding the short-term and long-term consequences of an action in its totality requires building a dynamic model to simulate the non-linear nature of the system. By knowing the ideology, it is believed that the models of BON and TLT demonstrate the ability to capture the critical time lags and relevant interactions among major variables. Also for those characteristics, it may be considered an innovative experimental model of system thinking in architecture. The two trajectories set as main outcomes, 'rethinking design' and 'rethinking construction', are crossing the boundaries of the discipline towards new scenarios. On one hand 
'rethinking design' involves reading and experimental design, exploring and understanding with a 'designing` mind; then planning tentatively, returning continuously to the reading in a mutual alternation which terminates invalid solutions. On the other hand, there is 'rethinking construction' by considering buildings as contextual elements, which means that every building must be considered in connection with its function, users, surroundings, and the environment because architecture is slowly shifting from a mechanical paradigm to a biologicalone.

Concerning the first target issue of BON; ethical standards; and social equity, TLT shows us that the formation of human identity can only be conceived as a social process and is triggered by the friction with the 'other'. Direct conflicts with diversity are almost impossible to negate because the formation of any identity awareness is created through the process of comparison, which means that each and every one of us identifies herself/himself through the recognition of what is different (Jenkins, 2004 and Auge, 1995). During the entire research process and through the specific playgrounds of TLT, the students were able to identify polarities of sameness and difference. This space of polarity exists in a social field where everyone is involved in a constant game of comparison and distinction in the quest for self-definition. Ethical standards imply social equity and vice versa.

The second target issue is the 'contextual impact' and 'holistic thinking'. BON states that the real project must convey a high standard of architectural quality in the way it addresses cultural and physical factors without forgetting the aesthetic impact which must not interfere with the surrounding environment. During an interview, Branzi (in Bombaci and Costanzo, 2011) says that the aesthetic of the field is the consequence of energies that appear through the diffusion of microprojects, sub-systems, commodities and services that are managed by design rather than by architecture. In rethinking design, BON capitalises processes that are capable of penetrating into the domestic interstices of everyday life. Additionally, drawing on Bourdieu (1984), Tonkinwise (2011) suggests that the success of a design intervention is often dependent upon its conformability or resonance with existing taste regimes. BON challenges the manner of cultivation of this expertise among young designers, allowing the opportunity to focus on the real capital of culture.

The third target issue is 'innovation and transferability of knowledge'. BON has proven to be of enormous interest to students, who have established innovative and lively interactive activities with the other participant Schools. As such, the initiative represents a highly positive learning experience and the kind of extra-curricular activity that would be eligible for the award of academic credit in line with the paper approved recently by Academic Council. The ambition of the group is also to develop its activity further and to mix research with teaching and design. However, these broader ambitions raise some questions BON believes is complicating different institutional plans.

BON believes that culture must be proposed as an operating system that guides the social organisation toward predefined temporary orders. Along with the iteration of the process, culture is the key to understanding complexity. In the model of BON the shift from design thinking to system 
thinking consists in designing platforms that can be used to integrate the iterative approach (system dynamics) and the challenge of self- organisation of socio-cultural systems into a comprehensive system methodology. In particular, the educational framework must change significantly to guarantee an empathetic civilisation for the next generations. Concerning future architects, they should be in the position to meet their needs (Fry 2009).

The following student feedback published at the End of Year Student Book 2011 of one of the Schools of TLT states that in the light of the first results the whole project of BON is already able to show the potentialities of a system thinking in design. The authors write: "We can do nothing to change this, so let's do something! [...] But the key result and achieved goal of this enterprise were the equipping of future architects in the tools of generative design in a spirit of optimistic realism. If even a fraction of the students in this workshop develops these themes in practice, many more people like the women of the DRC will be helped. One of the most valuable times in my education!”

\section{References}

Andreotti, L., Costa, X. (1996) Theory of the dérive and other Situationist Writings on the City, Barcelona: Actar.

Augé, M. (1995) Non-Places: Introduction to an Anthropology of Supermodernity, London: Verso.

Bassanese, S., Espanyol, A., \& Rodeghiero, B. (2012) Build Our Nation International Project: An Innovative Educational Model for Systems Thinking in Design, INTED2012 Proceedings, 4849-4858.

Bauman, Z. (2007) Liquid Times. Living in an Age of Uncertainty, Cambridge: Polity Press.

Bombaci, G., Costanzo, M. (2011) The Neutral Field: 2A+P/A interviews Andrea Branzi, San Rocco Islands 3, 147-153.

Bourdieu, P. (1984) Distinction: A Social Critique of the Judgment of Taste, Nice, R. (trans.) Boston: Harvard University Press.

Branzi, A. (1975) Radical Notes. Casabella 399(3), 10-14.

Brown, T. (2009) Change by Design: How Design Thinking Transforms Organizations and Inspires Innovation, New York: Harper Collins.

Charnley, F., \& Lemon, M. (2011) Exploring the Process of Whole System Design. Design Studies, 32(2), 156-179.

Peter, C. (1981) Systems Thinking, Systems Practice, Wiley.

Coleman, J. (1988) "Social Capital in the Creation of Human Capital". American Journal of Sociology Supplement 94: S95-S120.

Corboz, A. (1985) Il Territorio come Palinsesto. Casabella 516(9), 22-27.

D’Anjou, P. (20110 An Alternative Model for Ethical decision-making in design: A Sartrean 
Approach. Design Studies 32, 45-59.

De Carlo, G. (2005) Le ragioni dell 'architettura. Milano: operaDARC Electa.

Dorst, K. (2011)The Core of ‘design thinking’ and its Application. Design Studies, 32(6), 521-553.

Friedman, Y. (1977) Ou commence la ville. Paris: UNESCO Etablissements humains et environnement socio-culturel.

Friedman, Y. (2000) Utopies realisable. Nouvelle edition. Paris: L`Eclat. Friedman, Y. (2003)

L`architecture de Survie: une Philosophie de la Pauvrete, Paris: L`Eclat.

Fry, T. (2009) Design Futuring: Sustainability, Ethics and New Practice, Oxford: Berg.

Galimberti, U. (2009) L'ospite inquietante. Il nichilismo e i giovani.

Milano: Serie Bianca Feltrinelli.

Ghrajedaghi, J. (2011) Systems Thinking. Managing Chaos and Complexity. A Platform for Designing Business Architecture, Burlington: Morgan Kaufmann, Elsevier.

Holland, J.H. (2006) Studying Complex Adaptive Systems. Journal of Systems Science and Complexity 19(1), 1-8.

Iacovoni, A. (2003) Game Zone: Playgrounds between Virtual Scenarios and Reality, Basel: Birkhauser.

Jenkins, R. (2004) Social Identity, London: Routledge. Price, C. (2003) Re: CP.

Basel: Birkhauser.

Rapoport, A. (1990) The Meaning of the Built Environment. A Nonverbal Communication Approach, Beverly Hills: Sage Publications.

Rapoport, A. (2009) Cultura, Arquitectura y Diseno, Barcelona: Edicions UPC.

Tonkinwise, C. (2011) A Taste for Practices: Unrepressing Style in Design Thinking. Design Studies 32(6), 533-545.

Wasserman, B., Sullivan, P. J., \& Palermo, G. (2000) Ethics and the Practice of Architecture, New York: Wiley.

Woolcock, M. (2000) Social Capital: Implications for Development Theory, Research and Policy. The World Bank Research Observer, 15(2), 225-249. 